Cómo citar este trabajo: Zornoza Gallego, C. (2019). Pautas de movilidad e impacto de la dispersión urbana: análisis de la provincia de Valencia a partir de nuevas fuentes de datos. Boletín de la Asociación de Geógrafos Españoles, 82, 2773, 1-26. http://dx.doi.org/10.21138/bage.2773

\title{
Pautas de movilidad e impacto de la dispersión urbana: análisis de la provincia de Valencia a partir de nuevas fuentes de datos
}

\author{
Mobility patterns and impact of urban sprawl: \\ analysis of Valencia province using new data sources
}

\author{
Carmen Zornoza Gallego \\ carmen.zornoza@uv.es \\ Departamento de Geografía \\ Universidad de Valencia (España)
}

\begin{abstract}
Resumen
El trabajo está dedicado a obtener parámetros de movilidad del área urbana de Valencia empleando datos geolocalizados de la red social Twitter. Los objetivos son dos: reconocer la escala de los desplazamientos según la frecuencia de visita y cuantificar el impacto que tiene la dispersión urbana en la longitud de los desplazamientos. La metodología parte de la detección del lugar de residencia de cada usuario, sobre la cual poder hacer el análisis de los desplazamientos. Una vez establecida la residencia, se clasifican el resto de posiciones de cada usuario en función a la cantidad de veces que se visita un lugar, distinguiendo entre lugares: frecuentes y ocasionales. De esta forma, se obtiene información sobre los espacios de vida habitual, en los que las estrategias de consumo de tiempo se ven reflejadas, y sobre los espacios de vida ocasional, donde los desplazamientos responden a una menor fricción por distancia. Finalmente, el impacto de la dispersión urbana en los desplazamientos se mide a través de las distancias que recorren los usuarios residentes en zonas urbanas continuas en comparación con las discontinuas. Los resultados confirman el impacto que la urbanización discontinua tiene en la movilidad con un aumento medio de $4 \mathrm{Km}$.
\end{abstract}


Palabras clave: movilidad; frecuencia de desplazamientos; urbanización discontinua; nuevas fuentes de datos.

\begin{abstract}
The aim of the paper is to obtain mobility parameters from the urban area of Valencia using geolocated data from Twitter. The objectives are: recognizing the scale of the movements depending on the visit frequency and quantifying the impact of the urban sprawl on the distances covered. Methodology starts locating the homes of Twitter users in order to use this position as the basis for the analysis. Once home location is obtained for each user, the rest of the positions are classified taking into account the number of times a place is visited. We distinguish between frequently and occasionally visited places. In this way, we observe habitual life spaces, where strategies of time consumption are reflected, and occasional life spaces, where movements are not so influenced by the distance. Finally, the impact of urban sprawl in the mobility is measured using distances covered by residents in discontinuous urban areas in comparison of residents in continuous urban areas. Results confirm the impact of discontinuous urbanization on the mobility with an average increase of $4 \mathrm{Km}$.
\end{abstract}

Key words: mobility; proximity; urban sprawl; new data sources.

\title{
1 Introducción
}

El estudio actual de las áreas urbanas toma como punto de partida la indefinición de sus límites. Los conceptos de "ciudad difusa" (Indovina, 1998) o "ciudad sin límites" (Nel.lo, 1996) definen el fenómeno que rompe con la antigua dicotomía campo-ciudad. Como argumentan Salom y Albertos (2010) se forma un nuevo modelo de ciudad dispersa a partir de la descentralización residencial y económica derivadas, entre otros factores, de las mejoras del sistema de comunicaciones que provocan una menor fricción de la distancia.

Las afirmaciones anteriores plantean un escenario urbano de partida en el que las ciudades se han expandido ampliamente en el territorio y sobre el cual es complejo fijar los límites de la misma. Miralles-Guash y Cebollada (2009) proponían que ya no son las variables socio-económicas clásicas las que definen la ciudad, sino los flujos individuales cotidianos de su población. Se toma aquí la movilidad como variable explicativa básica sobre la que estudiar las áreas urbanas. Pero no sólo la movilidad modela la ciudad, también a la inversa. El "binomio imperfecto" fue como Miralles-Guash (2002) definió la relación entre la ciudad y la movilidad, describiendo la dependencia entre ambas y la ineficiencia de separar el urbanismo (desarrollado por arquitectos y planificadores) del trasporte (gestionado principalmente por ingenieros). 
El hecho de tomar la movilidad como parámetro para definir y estudiar las áreas urbanas resulta especialmente interesante para conocer cómo se han expandido. Para ello, es necesario introducir el concepto de tiempo dedicado a los desplazamientos. Esta perspectiva fue la que tomó Zahavi (1974), quien, bajo el encargo del Departamento de Transporte Estadounidense, realizó un análisis robusto sobre los hábitos de desplazamiento y el tiempo dedicado a los mismos. Hasta ese momento la mayoría de los modelos para evaluar el tráfico consideraban que un aumento en la velocidad de los desplazamientos impactaba directamente en una disminución del tiempo del viaje. Esto hacía que la evaluación coste-beneficio entre la mejora red de infraestructuras y el ahorro de combustible reportara un balance positivo. Pero el autor pudo verificar su hipótesis principal, centrada en la estabilidad de la cantidad de tiempo dedicado a los desplazamientos. Observó que el tiempo que las personas utilizaban para sus desplazamientos era relativamente constante (formalizado en 1h), lo que supuso un cuestionamiento absoluto de los modelos anteriores. Señaló entonces que el aumento de las velocidades ofrecidas por mejoras en los sistemas de transportes no se utilizaba para disminuir el tiempo que los ciudadanos dedicaban a sus desplazamientos, ni su coste, sino que se traducían en un aumento de la distancia recorrida y/o en más viajes.

La hipótesis de que los humanos desde épocas antiguas hayamos utilizado una cantidad de tiempo relativamente constante para los desplazamientos habituales es complicada de verificar por la falta de datos. Pero investigadores como Marchetti (1994) o Schafer \& Victor (2000) sí estimaron que una hora diaria es consistente con el comportamiento de las comunidades rurales modernas en vías de desarrollo y con la extensión de las zonas agrícolas intensivas alrededor de los pueblos rurales en Europa.

En la actualidad los registros sí permiten hacer un seguimiento detallado de esas dinámicas. Como describe Pumain (2003) los tiempos medios de desplazamientos habituales en distintos países son relativamente similares: en Hungría y Francia 55 m, en Inglaterra $1 \mathrm{~h} 24 \mathrm{~m}$, en Bélgica $1 \mathrm{~h} 23 \mathrm{~m}$, o en Dinamarca $1 \mathrm{~h} 15 \mathrm{~m}$. Estas observaciones proponen que, si la cantidad de tiempo que las personas dedican a sus desplazamientos es relativamente constante, de la velocidad a la que pueden trasladarse dependerá el espacio que puedan abarcar diariamente. Se toma entonces la velocidad de los transportes como una de las variables que permite explicar la expansión de las ciudades.

Mientras los desplazamientos a pie modelaron las ciudades, la compacidad y la mezcla de usos eran valores necesarios porque favorecían la accesibilidad. Según Newman et al. (2006), la mayoría de ciudades peatonales no sobrepasaron los 5-8 Km de diámetro. Marchetti (1994) analizó desde la perspectiva antropológica del ser humano su relación con los desplazamientos y señaló que las dimensiones de las ciudades pre-ferrocarril no sobrepasaban los $5 \mathrm{~km}$ de diámetro, coincidiendo con la distancia que puede recorrerse a pie en una hora. 
La llegada de ferrocarriles y tranvías impactó en el crecimiento de la ciudad sobre el trazado de las líneas, alcanzando distancias entre 20-30 Km. En el caso de la ciudad de València, la implantación de redes ferroviarias dio como resultado una convivencia de dos tipos de ciudades, la ciudad peatonal y la ferroviaria. El tipo de figura resultante fue descrita por Geddes en 1915 (en Bretagnolle, 2009) como "un pulpo", con una parte central, que se puede recorrer a pie, y los tentáculos que evolucionan sobre el trazado de las vías de ferrocarril. Las líneas y, específicamente las paradas o apeaderos, ejercían un efecto centralizador sobre la trama urbana.

La adopción del coche como modo de transporte mayoritario, junto con la masiva ampliación de la red viaria, produjo una transformación extensiva en la forma urbana. Las distancias se ampliaron hasta los 50 km, pero sin ningún efecto centralizador. Como dijo Merlin (1991, p. 427): "Mientras el transporte público tiene un efecto centralizador al reforzar su accesibilidad hacia el centro, las carreteras tienen un efecto descentralizador: cualquier punto en se convierte en un nodo absolutamente privilegiado". Surge entonces la denominada "ciudad del automóvil", que se extiende en el espacio produciendo una reescalarización de la realidad urbana caracterizada por la dispersión, la especialización funcional y la segregación social (Nel·lo, 2002).

Ahondar en el conocimiento de la movilidad de un área urbana actual resulta tan interesante como complejo. El estudio de los recorridos habituales de la población no es tarea sencilla ya que, al tamaño de la muestra y la complejidad de la obtención de datos, se suma lo apuntado por Kesselring (2006) sobre que la movilidad crece y se diversifica, siendo los flujos cada vez más numerosos y dispersos en el espacio y en el tiempo.

Hasta la fecha, es la movilidad laboral la que ha acumulado mayor número de estudios, ya que es el tipo de movilidad más estructurada de todas las que se producen en el ámbito metropolitano, repetida de forma cotidiana y con trayectorias y horarios relativamente fijos (Charron, 2007). Las aproximaciones al espacio metropolitano que dibuja este tipo de movilidad son frecuentes, ya que sus características hacen que la recogida de datos sea más sencilla. Obtener información de otros tipos de movilidades, hasta la fecha, era dependiente de la realización de encuestas específicas. En la actualidad es posible integrar otro tipo de actividades empleando datos provenientes de las nuevas tecnologías.

La proliferación de este tipo de datos en los últimos años se ha convertido en recurso de gran valor para las ciencias que estudian los comportamientos humanos. En las últimas dos décadas las nuevas tecnologías han pasado a formar parte de la cotidianeidad de la sociedad. El uso de teléfonos móviles, tarjetas de crédito, redes wifi, navegadores... crea un rastro digital con el que es posible trazar múltiples facetas de la vida de millones de individuos. El almacenamiento de esta información supone la mayor base de datos sobre el comportamiento humano que se haya obtenido jamás. 
Las ciencias sociales comienzan a generar información significativa a partir de estos registros digitales. Específicamente, las ciencias que estudian comportamientos humanos en relación con el territorio pueden nutrirse también de estas fuentes, ya que existen registros datos espaciales asociados. Según apuntan Li et al. (2016), existe un gran cambio en la disponibilidad con los datos geoespaciales, ya que hasta hace poco se producían bajo demanda y con un alto coste para las administraciones o empresas, mientras que actualmente la mayor parte de los mismos están siendo creados por cualquier persona a través del uso de su smartphone.

Esta componente espacial es una variable diferencial que puede resultar clave en el estudio de múltiples problemáticas y por supuesto es indispensable al trabajar con procesos territoriales. La movilidad cotidiana es uno de ellos, ya que estos registros además de contener datos sobre múltiples esferas de la vida diaria de sus usuarios se producen en tiempo real.

La dinámica diaria de una ciudad y su relación con los usos del suelo ha sido abordada por GarciaPalomares et al (2018). En este estudio emplearon datos Twitter para comprender cómo los usos del suelo influyen en la distribución de la población por periodos temporales. El análisis de datos provenientes del uso de la tarjeta de transporte inteligente ha permitido también explorar dinámicas de diferentes ciudades. Munizaga et al (2010) combinaron esta información con callejeros y líneas de autobús para inferir los orígenes y los destinos de los pasajeros, aplicando esta información a la tarificación de zonas de transporte.

La movilidad orientada a la actividad turística recoge también importantes aportaciones en el uso de nuevas fuentes de datos. Salas-Olmedo et al. (2018) emplean Panoramio, Foursquare y Twitter para inferir su actividad principal, relacionando las plataformas, por orden, con: hacer turismo, consumir, estar conectados. El análisis de clústeres espaciales según las actividades turísticas permite localizar los espacios turísticos multifuncionales y los que están especializados en alguna de ellas. En este campo también Gutiérrez-Puebla et al. (2017) analizaron los listados de Airbnb y fotografías geolocalizadas para conocer la distribución espacial de los alojamientos de esta plataforma en comparación con la oferta hotelera. Observaron cómo los alojamientos de Airbnb se encuentran más próximos a las principales atracciones turísticas, mientras que los hoteles predominan en áreas más periféricas. Los resultados permitieron establecer la relación entre el aumento de precios para residentes y la expansión de la plataforma en ciertas áreas de la ciudad.

El presente artículo persigue aportar información sobre la movilidad en la provincia de Valencia a partir del estudio de flujos individuales derivados de datos geolocalizados de la red social Twitter.

La primera de las cuestiones relativas a la movilidad que se propone en este artículo es reconocer la estrategia de sus habitantes para localizar sus actividades. Partimos aquí de la observación realizada por Miralles-Guash (2011), quien afirma que las áreas metropolitanas están inmersas en dos dinámicas territoriales contrapuestas y complementarias. La primera es una ampliación de la 
ciudad real, a través de la descentralización territorial y de la especialización funcional del entorno, y la segunda, un fenómeno de intensificación del barrio, asociado a la búsqueda de proximidad como valor en alza (Serra, 2007). Ambos procesos se revelan como complementarios en términos temporales, ya que el tiempo es un recurso limitado cuya gestión y control es percibido como elemento ligado a la calidad de vida (Durán, 2007). El objetivo es examinar la posible existencia de esta doble dinámica territorial en el Área Metropolitana de València, aunque con una restricción clara. En la afirmación realizada por Miralles-Guash (2011) se incluye un componente comparativo de dos momentos temporales con los conceptos de "ampliación" e "intensificación". La fuente de datos con la que se trabaja en este artículo, Twitter, comenzó a ser almacenada en 2016, por lo que el periodo temporal es corto para poder plantear un estudio fundamentado en términos comparativos. Nos limitamos entonces a plantear un análisis de la movilidad actual que, a partir de la frecuencia, la cantidad de desplazamientos y las distancias recorridas permita hacer una lectura de las citadas dinámicas en términos similares.

El primer análisis se centra en calcular las distancias recorridas por los usuarios, haciendo una distinción entre la frecuencia de visita del destino, tratando con dos tipologías de lugares: frecuentes y ocasionales. De esta forma seremos capaces de estudiar el impacto de la proximidad y de los desplazamientos más largos en función a la regularidad de los destinos.

El segundo de los análisis realizados versa sobre el impacto que tiene la urbanización discontinua en el aumento de la movilidad diaria, en comparación a la movilidad de personas residentes en zonas de urbanización continua. Los términos de zonas urbanas continuas y discontinuas se emplean en correspondencia con la fuente de datos empleada, CORINE Land Cover. De esta manera, se pretende relacionar la tipología de la forma urbana con las distancias recorridas, contribuyendo a cuantificar el impacto que la forma de la ciudad tiene en la movilidad, y con ello sus externalidades negativas. La dependencia del vehículo privado para los residentes en zonas dispersas es una característica común. Aunque en este trabajo no se analice el modo de desplazamiento sí se relacionará la tipología de residencia con las distancias recorridas. Según señaló la Agencia Ambiental Europea, las causas de los elevados niveles de contaminación en las ciudades se encuentran en su propia forma (European Environmetal Agency, 2006).

Múltiples investigaciones se han orientado a conocer el impacto que tiene sobre la movilidad el tipo de ciudad. Entre ellos Norman et al (2006) realizaron un estudio sobre dos áreas de Toronto (Canadá), comparando la energía y la contaminación que producen las urbanizaciones de alta y baja densidad. Calcularon el uso de energía asociado a los materiales de construcción, obras y medios de transporte de cada una de las tipologías. Obtuvieron que entre el 40-60\% de las emisiones son fruto del transporte. Y que los residentes de baja densidad emiten 4 veces más 
contaminantes. Rajamani et al. (2003) demostraron que en Portland (EEUU) la mezcla de usos y la alta densidad favorecen los desplazamientos peatonales para desplazamientos no laborales.

Sobre los costes del Urban Sprawl para la Comunitat Valenciana ha trabajado recientemente Gielen (2016), aunque bajo la perspectiva de los impactos monetarios para la administración pública. Este trabajo ha dado un vuelco a la percepción de que la urbanización supone un impacto positivo para los consistorios municipales, poniendo el foco en los crecimientos dispersos. Las administraciones consideraban que los rendimientos monetarios provenientes de la actividad urbanística eran la única variable en la ecuación, propiciando un ambiente favorable mayoritario a los amplios crecimientos urbanos. Pero tras el estudio realizado, el autor es capaz de cuantificar que el aumento de la dispersión municipal supone un incremento marginal del 21,5 al 24,2\% del gasto corriente anual de los municipios.

El ámbito de estudio sobre el que se desea obtener información es el área metropolitana de Valencia, pero se decide ampliarlo a toda la provincia por diversos motivos. El primero de ellos es comparar la información y los resultados de zonas que deben responder a dinámicas diversas (interiores, litorales, metropolitanas...). El segundo es obtener información sobre cierto tipo de actividades que tal vez se expanden más allá del territorio metropolitano. Las dificultades señaladas para definir las áreas urbanas, hacen que ampliar el área de estudio resulte útil para no constreñir el ámbito a áreas delimitadas bajo otros parámetros. Finalmente, también permite dejar abierta la posibilidad de plantear un estudio donde el espacio urbano sea abordado desde una perspectiva dinámica.

El trabajo aquí presentado forma parte de una investigación más extensa de la que ya se han publicado los primeros avances, relativos al proceso de filtrado de datos y a la propuesta metodológica para la detección de residencias (Zornoza \& Salom, 2018). En la citada publicación también se estudian los patrones de uso de la plataforma y se validan los resultados de la detección de residencias empleando análisis cualitativos y cuantitativos. El avance actual de las investigaciones ha permitido detectar nuevos filtros y nuevas cuestiones metodológicas que mejoran los resultados. Con la finalidad de presentar aquí un artículo auto contenido de buena comprensión, se especificarán brevemente las características principales de las investigaciones anteriores junto con las mejoras señaladas.

En resumen, el artículo se estructura de la siguiente manera: (1) análisis de la fuente de datos, (2) breve revisión metodológica, (3) cuantificación de las distancias medias recorridas en función al tipo de actividad (frecuente, ocasional), (4) cuantificación de las distancias medias recorridas en función al tipo de tejido urbano (continuo, discontinuo), (5) conclusiones. 


\section{Fuentes de datos}

La fuente principal de información es la red social Twitter. Su accesibilidad y la disponibilidad de datos georreferenciados permiten plantear un estudio de base territorial. Las ventajas respecto a fuentes tradicionales, como el censo de población, son: la posibilidad de bajar de la escala municipal, hacer un seguimiento individualizado de los desplazamientos, obtener una visión dinámica del área y registrar múltiples actividades de sus usuarios. Estas características permiten obtener información sobre los desplazamientos en el área y sobre los espacios de vida de sus habitantes.

Es necesario analizar las implicaciones de esta gran base de datos humana no sólo en términos de oportunidad, sino también en términos críticos que permitan crear investigaciones fundadas en una base sólida. A la necesidad de desarrollar mecanismos de análisis, almacenamiento y gestión de la información eficaces para el volumen en cuestión, se le suma la necesidad de profunda reflexión sobre la conveniencia de los datos para su aplicación al estudio de un proceso determinado, así como potentes mecanismos de control. Resultados al respecto se encuentran en Zornoza y Salom (2018).

El requerimiento de un análisis sólido de los propios datos se hace patente al entender la forma en que se producen. Los sujetos que generan los datos (las personas) los crean con una finalidad que no concuerda con la que luego pretende utilizarse en la investigación. Es decir, por ejemplo, una persona realiza un pago con una tarjeta de crédito con la finalidad de obtener un producto sin emplear dinero efectivo, sin más. Posteriormente, se quiere emplear ese rastro digital para producir información derivada (estudios de mercado, movilidad, influencia de áreas comerciales, empleo de tiempo...), que nada tiene que ver el motivo por el cual se realizó la transacción inicial. En este sentido, la transformación de los datos Twitter a información de movilidad requiere de un amplio proceso de filtrado y de la elaboración de una metodología adecuada.

La red social Twitter pone a disposición libre a través de su API un porcentaje de los tweets publicados en un área seleccionada, que pueden descargarse a tiempo real. En nuestro caso se seleccionan aquellos que han sido previamente georreferenciados por los usuarios ya se empleará la posición de los mismos como variable esencial de análisis. La muestra actual cuenta con un total de 602 días, 62339 usuarios y 580173 tweets.

La conveniencia de la base de datos empleada para estudiar el problema planteado debe ser el punto de partida de la investigación. Para el caso de Twitter, según se detalla en Zornoza y Salom (2018), existe un claro sesgo por edad, teniendo en cuenta que únicamente el $5 \%$ de la población mayor a 55 años tiene una cuenta abierta en Twitter. También existe un sesgo para los menores de 13 años, que no tienen acceso a la plataforma. En cambio, se señala como la muestra es muy amplia hasta los 44 años, con un 47,7\% de personas que tienen una cuenta abierta en Twitter. 
Existe también un pequeño sesgo de género en todos los rangos de edad, en el que las mujeres están infrarrepresentadas respecto a los hombres (46\% frente a $54 \%$ ).

Las limitaciones observadas impactan en los estudios actuales, pero a su vez son la muestra de que en el futuro se verán mitigadas por el envejecimiento de sectores de la población en el que el grado de penetración tecnológica es hoy una realidad. Esta es una de las mayores potencialidades prospectivas de los estudios basados en información proveniente de las nuevas tecnologías.

Se plantea así un análisis cuyos resultados están referidos a los sujetos que, por edad, potencialmente presentan las mayores movilidades. La movilidad de los menores y de la población de más edad, es menor, y además con unas características de accesibilidad distintas que deben ser abordadas acorde a sus necesidades. Los resultados que se obtienen en este trabajo serán entendidos bajo la casuística de máxima movilidad.

Otra de las necesidades a cubrir en el análisis de los datos es conocer qué información contiene un tweet geolocalizado para ser explotado convenientemente según las características del estudio. Los tweets geolocalizados permiten realizar un seguimiento individualizado de los trayectos ya que contienen un identificador único para cada usuario. Además, las coordenadas geográficas, la fecha y la hora en que se produce cada tweet, constituyen la información básica para modelar un patrón espacio-temporal.

\section{Metodología}

La metodología empleada consta de tres etapas fundamentales: (1) filtrado de datos, (2) clasificaciones de lugares, (3) cálculo de distancias recorridas. La primera etapa, referida al filtrado de datos, se encuentra ampliamente desarrollada en Zornoza y Salom (2018), aquí se especificarán las características básicas para permitir una buena comprensión del trabajo. La segunda etapa clasifica tres tipologías de lugares: residencias, lugares frecuentes y lugares ocasionales. La detección de residencias también fue abordada en la publicación anterior, mientras que las demás tipologías se desarrollan por primera vez en este trabajo. La tercera etapa, que versa sobre el cálculo de distancias también es una nueva aportación. Cada una de las etapas se aborda de forma diferenciada en los apartados siguientes.

\subsection{Filtrado de los datos:}

El filtrado de datos, centrado en obtener información de movilidad de los usuarios residentes en el área, consta correlativamente de las siguientes etapas:

- Filtrado de sensores: se eliminan aquellos usuarios cuyo comportamiento esté relacionado con un sensor (estaciones meteorológicas, de tráfico...). Estos usuarios se detectan a través de la fuente del tweet, ya que suelen emplear plataformas propias con muy pocos usuarios. Se 
calcula la ratio de tweets por fuente y usuario y se considera un sensor cuando una misma fuente tiene un pequeño número de usuarios frente a un gran número de tweets.

- Filtrado de posiciones no significativas: estas posiciones se producen cuando un usuario geolocaliza su tweet seleccionando un espacio poco preciso para la escala del estudio. Por ejemplo, si se quiere analizar un ámbito intra-metropolitano las localizaciones a nivel provincial o de comunidad autónoma ("Valencia" o "Comunitat Valenciana") no son válidas. Para evitarlas se realiza un conteo de las veces que la misma posición se repite para usuarios distintos. Los valores más altos de esta ratio dejan entrever estas posiciones, aunque se entremezclan con los lugares más frecuentados del área, por lo que se requiere una discriminación cuidadosa de estos puntos.

- Filtrado de turistas: se consideran turistas aquellos usuarios que han hecho uso de la plataforma en el área durante un periodo de tiempo menor a 60 días. A partir de este tiempo se consideran residentes.

- Filtrado de usuarios sin movilidad suficiente: los usuarios deben mostrar un mínimo de 5 posiciones diferentes para poder recabar datos de movilidad sobre sus perfiles.

- Filtrado de tweets por comportamiento: ante ciertos eventos importantes para los usuarios (deportivos, políticos, personales...etc.) se produce un uso intensivo puntual de las redes sociales. Para evitar la influencia de estos eventos todos los tweets generados por el mismo usuario en menos de 24 h en un radio de 100 m son contabilizados como una única posición.

\subsection{Detección del lugar de residencia, lugares frecuentes y lugares ocasionales}

La metodología empleada para la detección de residencias se encuentra específicamente desarrollada en Zornoza y Salom (2018). En el citado trabajo se tomó como base la investigación de Jurdak et al (2015), quienes observaron una alta correlación entre la residencia y el lugar desde el que frecuentemente se twittea. Siguiendo esta idea, la residencia se establecía en el lugar donde más tweets se realizaban, denominado posición dominante.

Tras este proceso, se seleccionan todos los puntos de los usuarios con residencia detectada y, con excepción de los lugares de residencia, se clasifican las demás posiciones. Se distinguirá ahora entre lugares frecuentes y ocasionales en función a la cantidad de veces que se visita un lugar, y no a la tipología específica de actividades a realizar. Esto resulta útil en el estadio actual de la investigación, ya que se analiza el hecho de que existe un desplazamiento, habitual u ocasional, pero obviando los motivos específicos. De esta forma, se dibujan dos escenarios: los espacios de vida habitual, en los que los ciudadanos realizan sus actividades comunes, y los espacios de vida ocasional, donde las actividades se consideran puntuales.

La forma de proceder es sencilla, se clasifican como lugares frecuentes aquellos que, no correspondiéndose con la residencia, han sido visitados en un mínimo de 3 ocasiones. Mientras 
que los lugares ocasionales los ocupan las posiciones que han sido visitadas en 1 o 2 momentos distintos.

\subsection{Cálculo de distancias recorridas}

Las distancias serán obtenidas sobre cada usuario único empleando una distancia euclidiana. Se ha decidido no realizar este análisis de distancias a través de la red viaria, ya que supondría afirmar que los desplazamientos no se producen sobre la red de metro o ferroviaria. Como no se incluye información modal del desplazamiento se ha considerado que la distancia euclidiana resulta más apropiada.

Se obtendrán las distancias medias diferenciando entre si el desplazamiento se produce a lugares frecuentes u ocasionales. El resumen de esta información de todos los usuarios ofrece una primera aproximación a las pautas generales de desplazamiento.

Finalmente, sobre el análisis anterior se añade una segunda distinción para reconocer a los residentes en zonas continuas y discontinuas. Para ello, se intersecta la localización de residencias con la información de usos del suelo del CORINE Land Cover 2011 y se calculan las distancias recorridas asociadas a cada tipología.

\section{Resultados: de las distancias recorridas calculadas}

Los resultados de la aplicación de los filtros se observan en la Tabla 1.

Tabla 1. Resumen de los filtrados

\begin{tabular}{|c|c|c|c|}
\hline & TIPO DE FILTRO & TWEETS & USUARIOS \\
\hline Inicial & & 580173 & 62339 \\
\hline Filtro 1 & Sensores & 484560 & 62298 \\
\hline Filtro 2 & Posiciones no significativas & 449245 & 58262 \\
\hline Filtro 3 & No residentes & 354604 & 17668 \\
\hline Filtro 4 & Comportamiento & 250109 & 17668 \\
\hline Filtro 5 & Movilidad reflejada & 181215 & 5505 \\
\hline
\end{tabular}

Leyenda: información inicial obtenida de datos de la API Twitter 2015-2018, información derivada obtenida a través del proceso de filtrado.

Fuente: elaboración propia 
Tras este proceso queda una muestra de 5505 usuarios, 181215 tweets y 84643 posiciones diferentes. Estos usuarios son considerados residentes y "trazables", es decir, que muestran una movilidad mínima de 5 posiciones distintas. Sobre ellos se comienza el proceso de detección de residencias, obteniendo un total de 4289. Más de mil usuarios considerados trazables inicialmente no han pasado el filtro final para localizar su residencia, basado en que la posición mayoritaria se haya repetido al menos 3 veces.

Las características de los usuarios sobre los que se detecta la residencia y sobre los que se van a obtener los patrones de movilidad se resumen en las siguientes figuras (Figura 1 y Figura 2).

Figura 1. Periodo de seguimiento por usuario

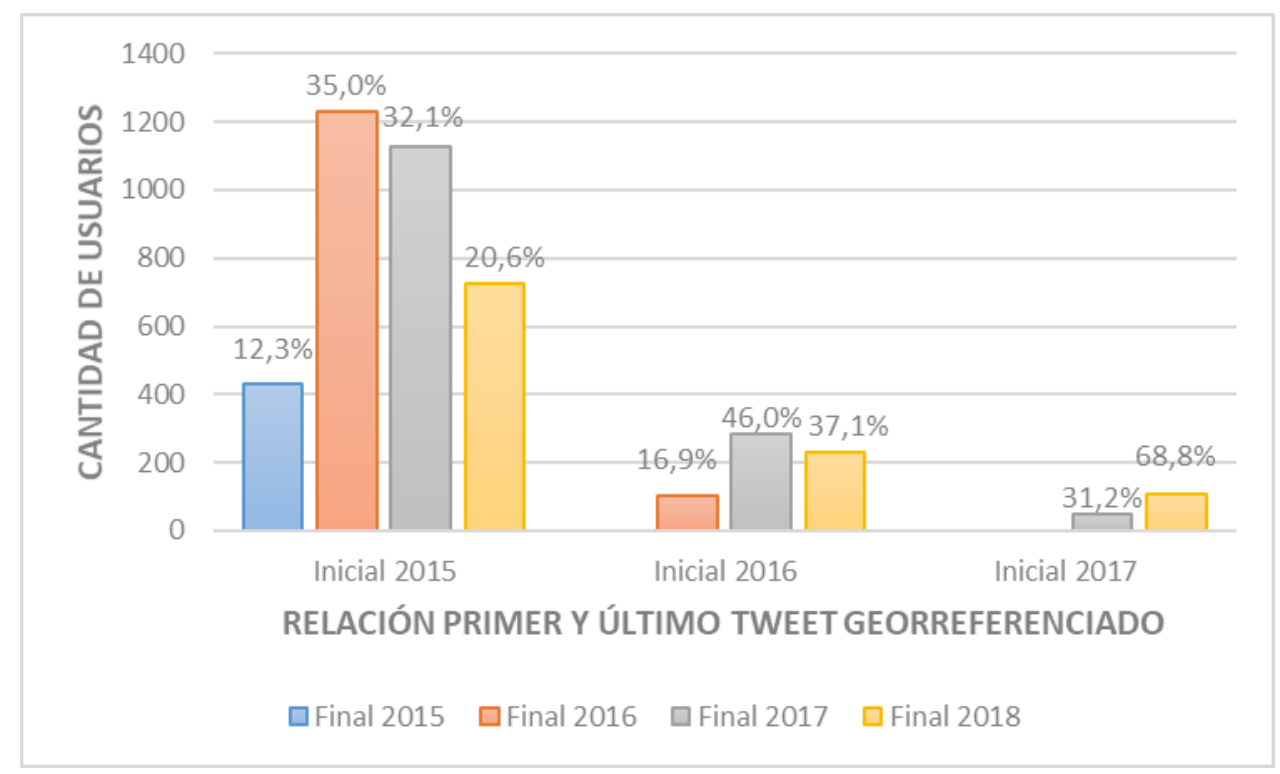

Fuente: elaboración propia

Los resultados que componen la Figura 1 muestran que la gran mayoría (82\%) de los usuarios con los que se calculan los parámetros de movilidad en el área de Valencia fueron captados por primera vez durante 2015. Sobre estos usuarios sólo un 12 \% terminó su actividad el mismo año y un $35 \%$ registró su último tweet georreferenciado en 2016. El 52,7 \% de estos usuarios han realizado algún tweet georreferenciado durante 2017 y 2018. Estos datos son indicativos de que, tras los filtrados pertinentes para capturar las personas residentes y con una actividad de tweets georreferenciados mínima (véase apartado 3.1.), la muestra se alarga considerablemente en el tiempo. Los usuarios que registran este tipo de actividad de forma continuada suelen estar activos amplios periodos.

También resulta muy interesante caracterizar la muestra con la cantidad de posiciones distintas que registra cada usuario. Es posible conocer así sobre cuantas posiciones se están calculando los parámetros de movilidad (Figura 2). 
Figura 2. Cantidad de posiciones diferentes por usuario

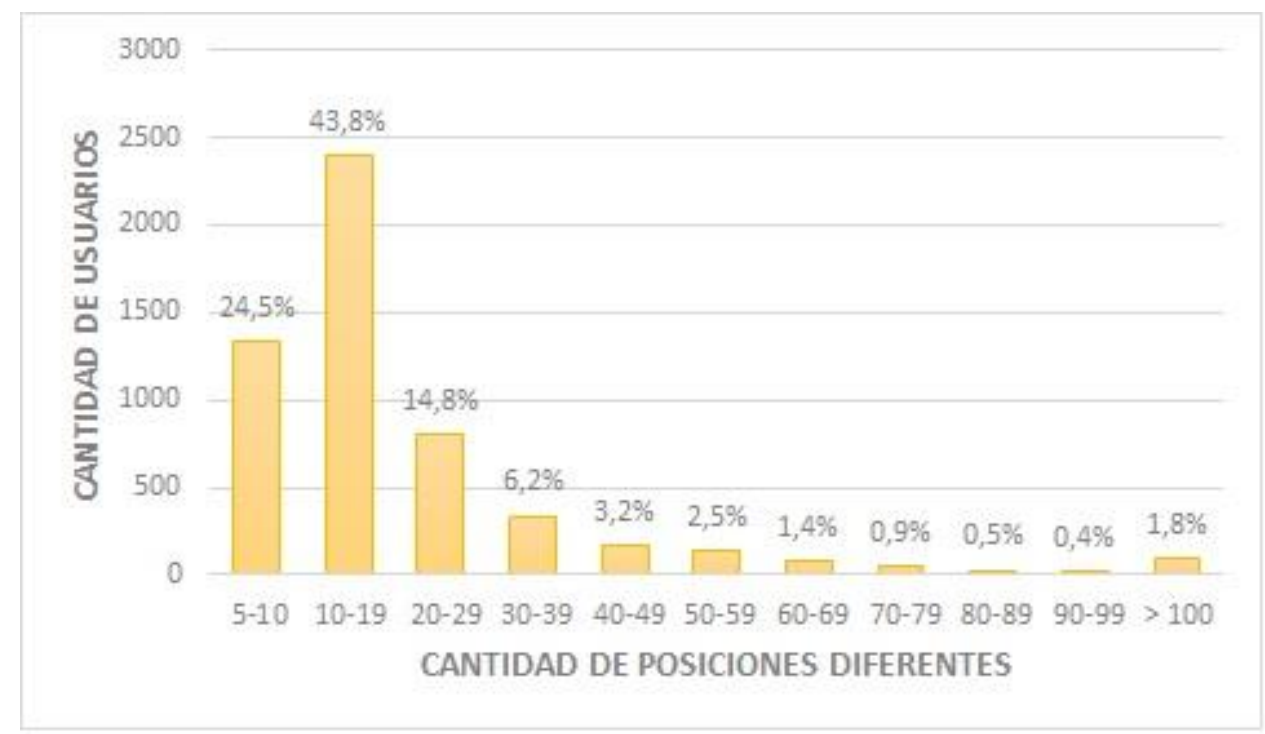

Fuente: elaboración propia

Las frecuencias observadas son indicativas del uso de tweets georreferenciados que realiza cada usuario, siempre teniendo en cuenta que no se trata de un usuario medio, sino de aquellos usuarios que han pasado los filtros pertinentes para este estudio. Los usuarios que componen la muestra, obligatoriamente, tienen un mínimo de 5 posiciones diferentes. De la información anterior se extrae que el $68,3 \%$ de los usuarios tiene entre 5 y 20 posiciones registradas y un $83,1 \%$ si se amplía hasta las 30 posiciones. Consecuentemente, el 16,9\% restante son usuarios que muestran más de 30 posiciones.

La última caracterización de la muestra se basa en la localización espacial de las residencias sobre la base municipal y las distintas franjas que establece la Estrategia Territorial de la Comunidad Valenciana (ETCV) (Figura 3). Estas franjas definen los tres grandes ámbitos territoriales sintetizando el conjunto de elementos urbanos, ambientales y paisajísticos que configuran el territorio valenciano (Directrices ETCV, p. 52).

Se observa en la figura anterior que la mayor aglomeración de residencias se localiza sobre la ciudad de Valencia y sus municipios contiguos. El área urbana morfológica trazada por el Institut de Gestion de l'Environnement et d'Aménagement du Territoire (2006) apoya la idea de que es sobre las áreas urbanas sobre las que la muestra contiene mayor cantidad de información y, por lo tanto, sobre las que los estudios basados en datos provenientes de la tecnología tienen mayor relevancia.

Es en los municipios asociados a ámbitos rurales, según clasificación de la ETCV, donde se detectan un máximo de 3 residencias e incluso municipios sin ninguna. Este resultado ilustra bien la validez de las fuentes de datos asociadas a las nuevas tecnologías, ya que su empleo es totalmente dependiente de las características del lugar de aplicación. Los factores que impactan en una 
infrarrepresentación del ámbito rural son: un peor acceso a internet, por la red de comunicaciones, y una menor penetración de la tecnología, debida a una población envejecida.

Figura 3. Localización de las residencias en su contexto territorial

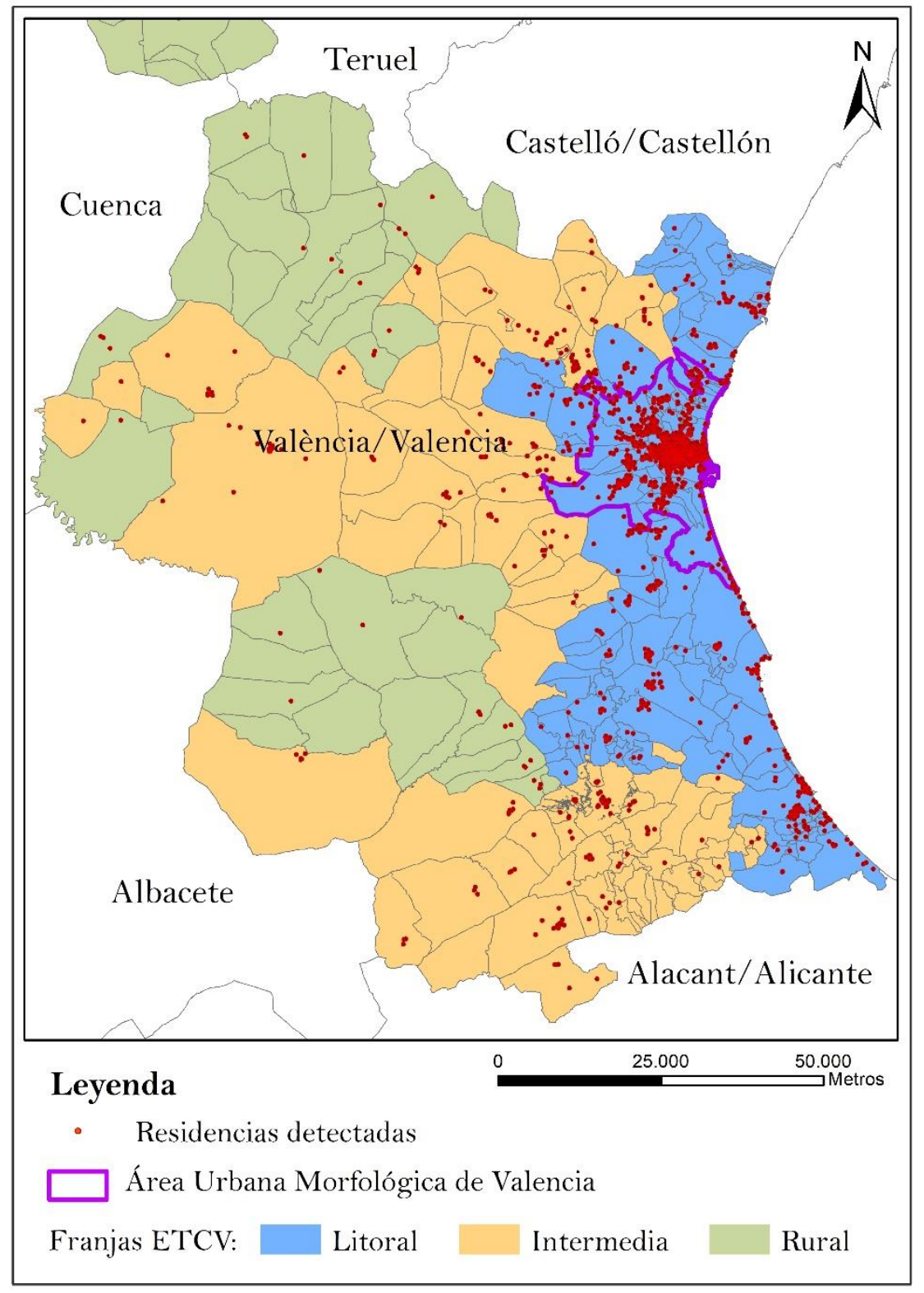

Fuente: elaboración propia

\subsection{Desplazamientos según destinos frecuentes y ocasionales}

A partir de las residencias se calculan las distancias medias recorridas a los lugares frecuentes y ocasionales. Se obtiene así una primera imagen de los procesos que se dan a cabo en el área (Tabla 2). 
Tabla 2. Distancias medias según frecuencias

\begin{tabular}{|c|c|c|c|}
\hline LUGAR & DISTANCIA MEDIA (M) & $\begin{array}{c}\text { TOTAL } \\
\text { POSICIONES }\end{array}$ & $\begin{array}{c}\% \\
\text { POSICIONES }\end{array}$ \\
\hline Frecuente & 10276,0 & 8573 & $10,7 \%$ \\
\hline Ocasional & 15643,1 & 71711 & $89.3 \%$ \\
\hline
\end{tabular}

Fuente: elaboración propia

La lectura más directa es que las distancias medias a lugares ocasionales son 5,4 Km superiores a las de los lugares frecuentes. Es decir, que se observa una tendencia de las personas a residir cerca de los lugares a los que acuden frecuentemente y/o a la inversa, a frecuentar los lugares cerca de su residencia. En la localización de los lugares frecuentes se refleja claramente el impacto de la proximidad. En cambio, los lugares ocasionales generan una movilidad de mayor distancia. Las estrategias de consumo de tiempo para realizar una actividad puntual, relacionando el tiempo con la distancia, no tienen tanto peso como en el caso de las actividades frecuentes.

Por otro lado, también es interesante observar como la cantidad de lugares frecuentes es menor que la de lugares ocasionales (10,7\% frente a un $89,3 \%$ ). El motivo se corresponde con la propia naturaleza de los datos, provenientes de una red social. Los tweets se generan con un patrón espacial y temporal distinto en función al tipo de actividad que se esté realizando. Como ya se detalló en Zornoza y Salom (2018):

Un lugar frecuentado habitualmente se repite en el espacio en varias ocasiones, aunque tenga menos posibilidades de generar un tweet cada vez que se acude. Un lugar poco frecuentado tiene más posibilidades de generar un tweet, pero no de repetirse espacialmente. Los lugares identificados de esta forma pueden ser vinculados a actividades de ocio, mientras que los que sí se repiten en el espacio fuera de casa serían considerados actividades cotidianas.

Se obtiene aquí un amplio registro de actividades ocasionales, como era de esperar, que nos acercan a una realidad urbana difícil de observar bajo otros registros, ya que se trata de actividades puntuales no estructuradas en el tiempo y en el espacio, sobre las que no existen estadísticas específicas.

Tras observar los valores medios, se requiere un estudio en mayor profundidad de la información de movilidad obtenida. Para ello, un análisis de la distribución de los valores (Figura 4) permite conocer la cantidad de desplazamientos en función a la distancia desde las residencias. 
Figura 4. Distribución de lugares frecuentes y ocasionales según distancia a la residencia

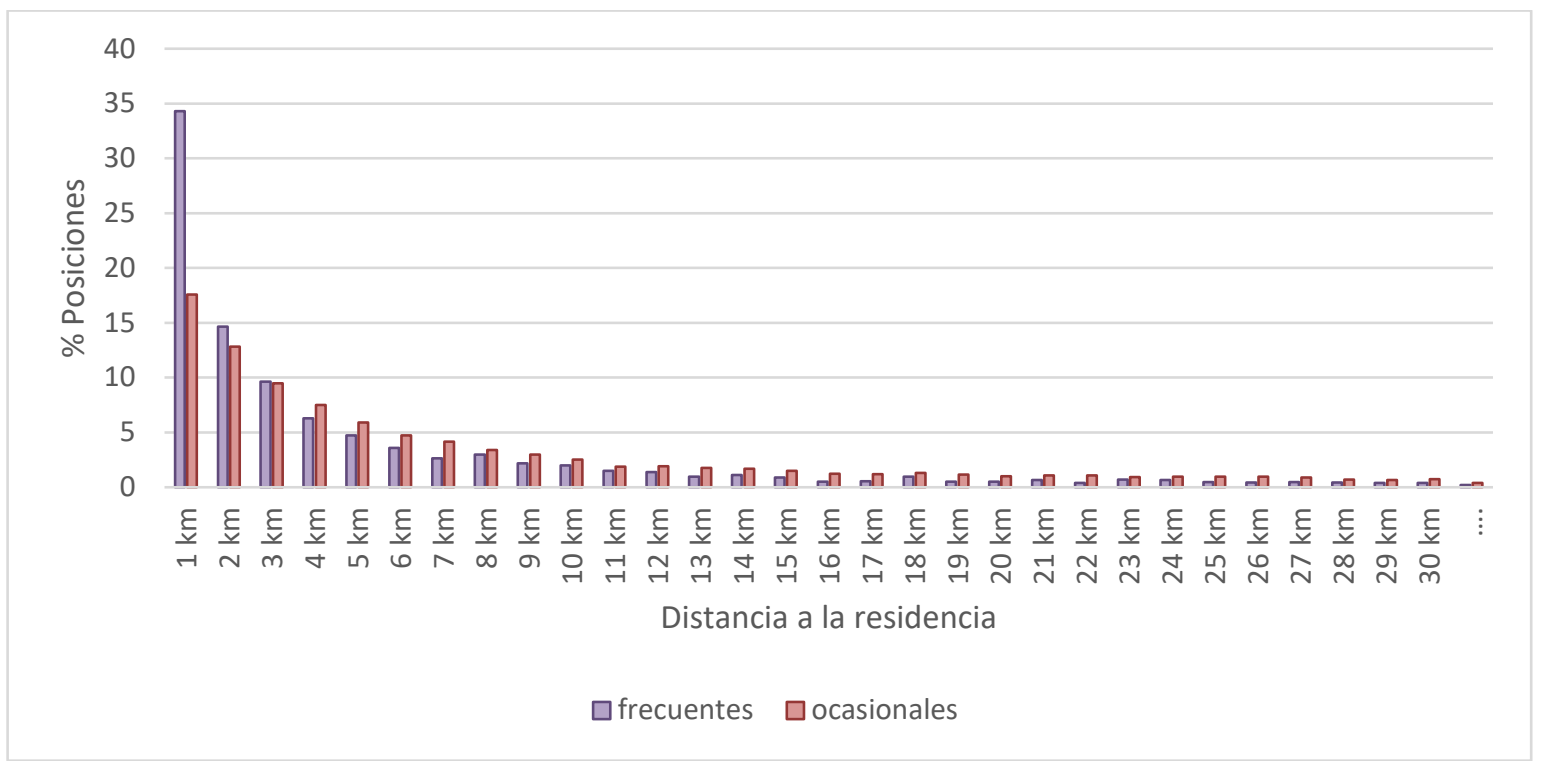

Fuente: elaboración propia

Se observa en la Figura 4 cómo las medias (obtenidas en la Tabla 2) se ven afectadas por valores extremos, pero que la gran mayoría de desplazamientos responden a la lógica de la proximidad. El $34,3 \%$ del total lugares frecuentes se encuentran a menos de $1 \mathrm{Km}$ de distancia del lugar de residencia, un $49,0 \%$ a menos de $2 \mathrm{Km}$ y un $69,6 \%$ a menos de $5 \mathrm{Km}$. La evolución de estos valores muestra que existe un alto número de lugares frecuentes cercanos al domicilio y una rápida disminución de estos lugares conforme las distancias aumentan. La primera lectura de estos resultados se relaciona con la denominada "Primera ley de la Geografía", acuñada por Tobler (1970): "Todo está relacionado entre sí, pero las cosas más próximas están más relacionadas que las distantes".

Tal y como se especificaba anteriormente, el trabajo pretende reconocer si la doble dinámica en la que están inmersas las áreas metropolitanas observada por Miralles-Guash (2011) se produce también en Valencia. Sin duda, la dinámica que apunta a la búsqueda de proximidad para realizar las actividades frecuentes parece verse bien refrendada con los valores obtenidos. Esta pauta es natural, ya que reduce el consumo de tiempo diario destinado a la movilidad.

La segunda, que apunta a unas dinámicas de movilidad que se extienden en el espacio, al ritmo de la ciudad difusa, son más complejas de observar, al producirse precisamente a distancias más diversas. Una característica que puede sustentar esta movilidad es la existencia del 30,4\% de los lugares frecuentes detectados a más de $5 \mathrm{Km}$ de la residencia. Estos $5 \mathrm{~km}$ marcan los límites habituales de ciudad a pie (Marchetti, 1994; Newman et al.,2006), por lo que, todo lo que se extienda más allá responde a una ciudad más amplia. 
Las distancias que se está dispuesto a recorrer para alcanzar lugares ocasionales son mayores que para el caso de las frecuentes con sólo un 17,6 \% a menos de $1 \mathrm{~km}$ de distancia de la residencia en contraposición con el 34,3\% de los frecuentes. Para alcanzar un porcentaje similar al de las actividades frecuentes a los $5 \mathrm{~km}(69,6 \%)$ en el caso de las ocasionales hay que desplazarse hasta los $10 \mathrm{~km}$. Se observa claramente que las actividades ocasionales se extienden más en el territorio, ya que las personas no las consideran dentro de las estrategias diarias de consumo de tiempo.

Finalmente, los datos mostrados adquieren todo su valor y potencial al plasmarlos en el territorio (Figura 5).

Figura 5. Distancia a lugares frecuentes y ocasionales
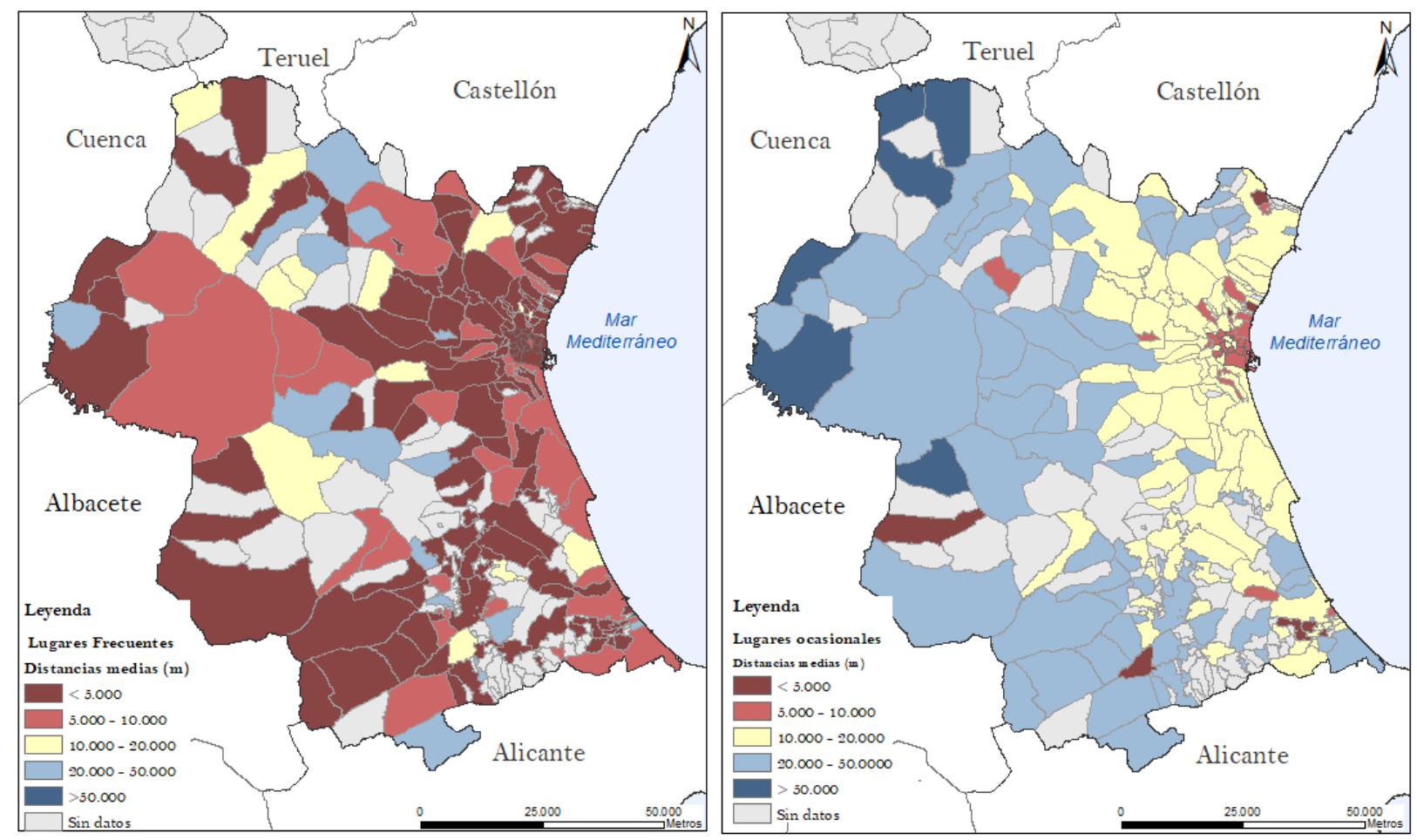

Fuente: elaboración propia

El mapa de la izquierda muestra las distancias medias a los lugares frecuentes a nivel municipal. Se obtienen los resultados esperados tras el análisis de la Figura 4, donde se señalaba que los lugares frecuentemente visitados se encuentran cerca de la residencia. Esto supone que no exista variación sustancial entre los distintos municipios de la provincia. No ocurre lo mismo en el caso de las actividades ocasionales plasmado en el mapa dela derecha. Aquí se observa que las distancias medias de los residentes en los distritos céntricos del municipio de Valencia son mucho menores a la de los municipios contiguos, y además que van aumentando en los municipios interiores. Se deriva de esto que en el área de Valencia la oferta de ocio se encuentra principalmente centralizada en la ciudad. 


\subsection{Desplazamientos según origen en tejido urbano continuo o discontinuo}

Pasamos ahora a la segunda cuestión planteada, destinada a conocer las diferencias en las distancias recorridas para los residentes en tejido urbano continuo y discontinuo (Tabla 3):

Tabla 3. Distancias medias según frecuencias y tipo de residencial

\begin{tabular}{|c|c|c|}
\hline LUGAR & $\begin{array}{c}\text { Distancia media }(\mathrm{m}) \\
\text { Tejido Urbano } \\
\text { continuo }\end{array}$ & $\begin{array}{c}\text { Distancia media }(\mathrm{m}) \\
\text { Tejido Urbano } \\
\text { discontinuo }\end{array}$ \\
\hline Frecuente & 8580,4 & 12347,5 \\
\hline Ocasional & 13034,2 & 17619,0 \\
\hline
\end{tabular}

Fuente: elaboración propia

Se constata que las personas que habitan en zonas urbanas discontinuas recorren 3767,1 m más que los residentes en zonas urbanas continuas para el caso de actividades frecuentes y 4584,8 m más para ocasionales. Estos resultados son información relevante para cuantificar el impacto de la urbanización discontinua en la movilidad y con ello en el gasto de recursos asociados a la misma. Para reconocer exactamente cómo se distribuyen estos desplazamientos se genera un gráfico con los lugares frecuentes (Figura 6).

Figura 6. Distribución de lugares frecuentes y ocasionales para zonas urbanas continuas y discontinuas

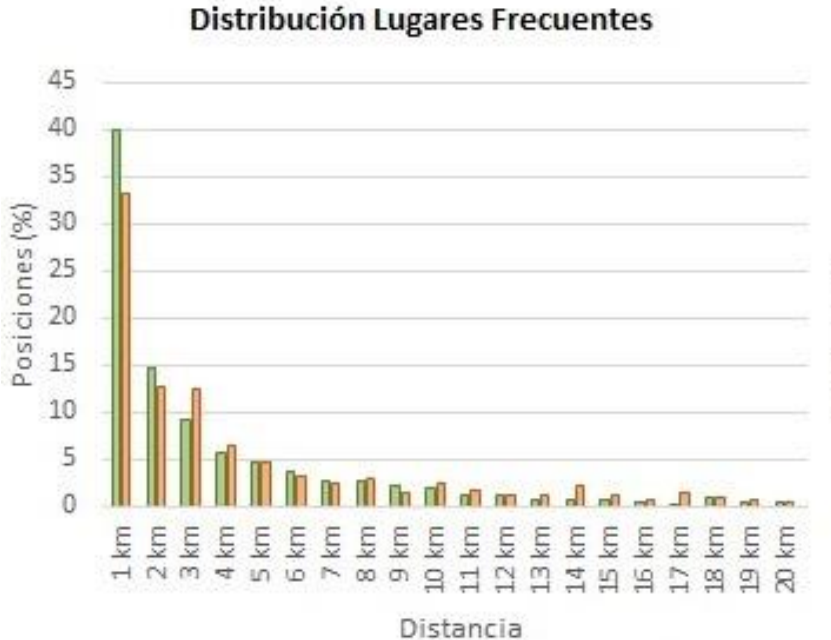

Distancia
Distribución Lugares Ocasionales

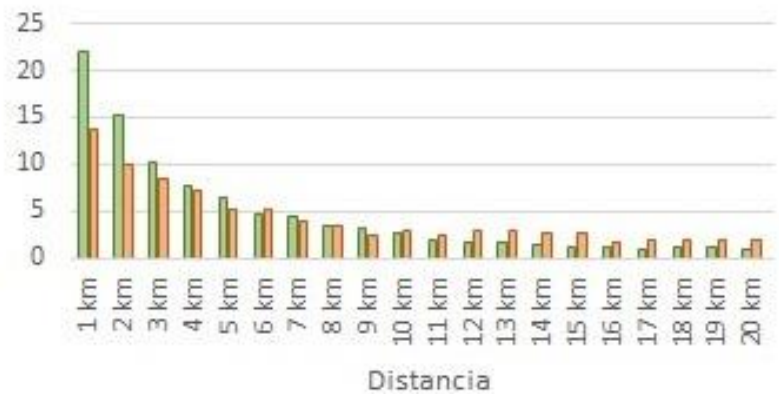

口urbano continuo $\square$ urbano discontinuo

Fuente: elaboración propia 
En las zonas urbanas continuas el 40,0\% de los desplazamientos frecuentes se realiza en el primer kilómetro, mientras que en el urbano discontinuo se reduce al 33,1\%. Rebasado el segundo kilómetro los desplazamientos en urbano discontinuo son porcentualmente mayores que los del urbano continuo. Estos resultados enfatizan cómo la forma urbana impacta directamente en la movilidad, generando mayor cantidad de desplazamientos lejanos al domicilio en las zonas urbanas discontinuas frente a las continuas. La argumentación llevada a cabo por Miralles-Guash (2011) aludiendo a la existencia de dos dinámicas territoriales complementarias se refuerza al interpretarla bajo estos resultados. En primer lugar, las dinámicas de proximidad o de intensificación de barrio se dan en mayor proporción en las zonas urbanas continuas, allí donde la mezcla de usos está más presente y posibilita un tipo de intercambios más cercanos. Rebasados los dos kilómetros, son los tejidos urbanos discontinuos quienes tienen mayor porcentaje de desplazamientos realizados, es decir, que son los responsables de generar más desplazamientos y más lejanos, teniendo mayor peso en la ampliación de la ciudad real.

En el caso de los lugares ocasionales, el 21,9\% de los desplazamientos en urbano continuo ocurren en el primer kilómetro, mientras que en urbano discontinuo el porcentaje baja al 13,8 \%. Las curvas que se dibujan en la Figura 6 resultan interesantes en su análisis, ya que, mientras la pendiente del urbano continuo disminuye rápidamente la del urbano discontinuo lo hace más suavemente, con una tendencia siempre superior desde los $9 \mathrm{Km}$.

Estos resultados son una primera aproximación al impacto que tiene en la movilidad del área urbana de Valencia la tipología de urbanización en la que se resida. Se observa la importancia de la morfología de la ciudad en los desplazamientos que genera, potenciando un tipo de desplazamiento u otro. El hecho de que en las zonas compactas se produzcan desplazamientos más cortos, supone directamente potenciar una movilidad más sostenible.

La expansión del espacio urbano, al igual que en muchas otras metrópolis, ha venido asociada al aumento de crecimientos residenciales de baja densidad. Al incremento de costes de infraestructuras, de mayor segregación social o de pérdida de espacios libres, se le añade una mayor dependencia del trasporte privado motorizado, que se traduce a su vez, en aumento de la congestión del tráfico, mayor consumo de energía y emisiones contaminantes (OECD, 2010; CEC, 2004).

\section{Conclusiones}

La investigación presentada muestra como Twitter es una fuente de datos válida para el estudio de pautas de movilidad diaria de las personas. La gran cantidad de datos representa su mayor potencial y, a su vez, su mayor problemática. La metodología de filtrado y de extracción de la información está orientada a obtener los mejores resultados facilitando los cálculos y los procesos. 
Las limitaciones generales de la fuente se basan en el sesgo de edad, siendo la población infantil y mayor de 55 años ampliamente infrarrepresentada. Esto supone que los resultados de este trabajo estén referidos a las personas de edad media, que potencialmente presentan las mayores movilidades.

El análisis de la muestra específica, con la que se obtienen los parámetros de movilidad de la provincia de Valencia, se ha centrado en: la cantidad de tiempo sobre la que se tiene registro de cada usuario, la cantidad de posiciones distintas que se han registrado y la localización espacial de las residencias. Se concluye que los residentes que forman parte del trabajo son usuarios de larga trayectoria en la red social Twitter, ya que más de la mitad de los que fueron captados por primera vez en 2015 seguían generando tweets georreferenciados durante 2017 y 2018, obteniendo así una muestra dilatada en el tiempo. Por otro lado, la cantidad de lugares geoposicionados de cada usuario es dependiente directamente de su actividad en la red social. La muestra recoge unas frecuencias medias entre 5 y 20 posiciones diferentes para el 68,3\% de los usuarios. La localización de residencias sobre el territorio indica que este tipo de estudios, que emplea datos provenientes de las nuevas tecnologías, son más propicios para ámbitos urbanos, donde la penetración de los dispositivos inteligentes y de las redes sociales es mayor que en el ámbito rural.

El hilo conductor del artículo ha sido la argumentación de Miralles-Guash (2011) sobre la existencia de dos dinámicas territoriales complementarias y contrapuestas, producto de la ampliación de la ciudad real, a partir de la descentralización territorial y la especialización funcional del entorno, y de la intensificación del barrio, en búsqueda de la proximidad. Las restricciones temporales de nuestra base de datos han imposibilitado el estudio comparativo anterior, pero sí ha sido posible realizar una lectura actual de dichas dinámicas.

Los resultados de movilidad se han estructurado en torno a dos cuestiones fundamentales: las pautas de movilidad según la frecuencia con que se realicen las actividades y el impacto de la dispersión urbana.

La diferenciación entre lugares visitados frecuentemente y lugares ocasionales ofrece una primera lectura consecuencia directa de la naturaleza de la fuente, donde únicamente el 10,7\% de los lugares se han considerado frecuentes, frente al 89,3\% de ocasionales. Más allá de esta caracterización, los resultados nos han acercado a comprender las diferencias entre los espacios de vida habituales y ocasionales. La movilidad que generan los lugares frecuentes es mucho más cercana que la asociada a los ocasionales. El 34,3\% de los lugares visitados frecuentemente se encuentran a menos de $1 \mathrm{Km}$ de la residencia y, en el caso de los ocasionales, este porcentaje quedaba reducido al 17,6\%. A su vez, las distancias medias recorridas desde cada residencia a los lugares ocasionales son $5 \mathrm{Km}$ superiores a las de los lugares frecuentes. Se hace patente que en los espacios de vida habitual la proximidad es una estrategia necesaria para minimizar el consumo 
de tiempo en los desplazamientos, mientras que en los espacios de vida ocasional los desplazamientos responden a una menor fricción por distancia. La transposición territorial de estos datos muestra una gran concentración de lugares ocasionales en el centro de Valencia, siendo reflejo de que la oferta de ocio se encuentra centralizada en la ciudad central.

La pretensión de cuantificar el impacto del tipo de tejido urbano en la movilidad ha resultado de especial interés. Para el tejido continuo se recorren distancias medias inferiores a las del discontinuo, con una diferencia de $3767,1 \mathrm{~m}$ para el caso de actividades frecuentes y 4584,8 $\mathrm{m}$ para ocasionales. Tras este primer resultado, los histogramas indican que en el primer kilómetro se producen el 40,0\% de desplazamientos frecuentes en las zonas urbanas continuas, en contraposición con el 33,1\% en urbano discontinuo. Los desplazamientos ocasionales siguen un patrón similar en el primer kilómetro, con un 21, 9 \% de los desplazamientos en urbano continuo y un $13,8 \%$ en el discontinuo.

Se concluye que los datos obtenidos pueden enmarcarse bien en la doble dinámica territorial analizada. La dinámica asociada a la proximidad ha sido evaluada a partir de los destinos frecuentes, donde el 69,6\% se encuentran a menos de $5 \mathrm{Km}$ de la residencia, haciendo que sea la proximidad la variable que impera en los intercambios y en el modelado del territorio. La dinámica contrapuesta, asociada a las largas distancias se ha evaluado teniendo en cuenta que el $30,4 \%$ de los desplazamientos frecuentes se realizan a una distancia mayor a $5 \mathrm{Km}$. Analizando los desplazamientos por tipología residencial, se observa que rebasados los primeros dos kilómetros son los tejidos urbanos discontinuos quienes generan mayor porcentaje de desplazamientos. Estos resultados pueden relacionarse con la diversidad de usos integrados en cada uno de los tejidos. En las zonas continuas la mezcla de usos posibilita desplazamientos cercanos, mientras que en las discontinuas la especificidad funcional es mayor, por lo que se requieren desplazamientos más lejanos para acceder a las actividades. La elección modal no ha sido un dato incluido en el análisis, aunque recorrer mayores distancias supone una mayor dependencia al vehículo privado. Además, la disminución de la densidad supone una menor eficacia de servicios públicos de transportes en tejidos urbanos discontinuos, suponiendo también un mayor uso potencial del transporte privado.

En definitiva, los datos presentados en este trabajo permiten cuantificar el impacto directo que la forma urbana tiene sobre la movilidad diaria de sus residentes. Más allá del estudio de caso, los resultados pueden extrapolarse al estudio de los impactos de las tipologías residenciales discontinuas, que son responsables de generar más desplazamientos y más lejanos, teniendo mayor peso en la ampliación de la ciudad real y en la sostenibilidad del área. Las implicaciones medio ambientales derivadas del aumento de la movilidad tienen su reflejo directo en una mayor cantidad de emisiones contaminantes y, con ello, una disminución de la calidad del aire. Las consecuencias para la salud de los ciudadanos son claras teniendo en cuenta las 38600 muertes prematuras en 
España durante 2015 que se asocian a este problema (European Environmental Agency, 2018). La importancia de la cuestión es creciente, y la aplicación de medidas para reducir estos niveles resulta compleja y controvertida, ya que impacta directamente en los hábitos de la población. El sector de la movilidad es uno de los que más se resiste a las políticas sostenibles (Næss, 2006).

Los resultados obtenidos pueden ser de utilidad en materia de políticas públicas. Aunque la reducción de las emisiones contaminantes sea posible con una elección modal sostenible de los ciudadanos, las políticas no pueden estar centradas únicamente en esta cuestión. Su efectividad se encuentra condicionada por la forma en la que crecen las ciudades que, como hemos visto, es responsable de modelar los desplazamientos. La discusión en este punto merece volver a la idea de la cantidad de tiempo relativamente constante que utilizamos los humanos dedicado a los desplazamientos (Zahavi, 1974). Aunque los motivos por los que las áreas urbanas se extienden de forma dispersa son múltiples, un principio básico es que se puede acceder a la ciudad central en un tiempo relativamente corto (alrededor de una hora ida y vuelta). Si tenemos en cuenta las dificultades del transporte público para servir áreas de baja densidad, las únicas velocidades que permiten estos desplazamientos se realizan empleando el vehículo privado. Por tanto, las políticas orientadas a mejoras en la red de trasporte público y a la concienciación de los ciudadanos por su uso son absolutamente necesarias, pero inefectivas si la ciudad sigue creciendo de forma dispersa. La necesidad de pensar y re-pensar las ciudades debe abordarse de forma integral, relacionando el urbanismo con todos los aspectos que tengan impacto en los ciudadanos: sociales, culturales, económicos y medioambientales. En definitiva, practicando una ordenación de territorio efectiva y en favor del interés general.

Sobre las líneas futuras de la investigación cabe señalar que inferir las características socioeconómicas de los usuarios en una segunda fase de análisis posibilitaría reconocer pautas de movilidad para los distintos grupos. Esta información permitiría ahondar en las características específicas de la movilidad observada, de forma que se detecten distintas necesidades a cubrir, así como dirigir las distintas políticas de movilidad sostenible hacia el público indicado.

Como punto final de las conclusiones, se considera importante abordar una reflexión sobre el uso de los datos provenientes de las nuevas tecnologías. Goodchild (2007) acuñó un término interesante con respecto a quién y cómo se generan los datos: "Citizens as sensors" (Ciudadanos como sensores). Si un sensor es un dispositivo que capta estímulos externos y responde en consecuencia, observamos cómo las personas que empleamos nuevas tecnologías en nuestras actividades diarias somos grandes receptores de estímulos que convertimos, generalmente a tiempo real, en datos. Como concepto resulta especialmente atrayente, ya que permite ahondar en el conocimiento de los comportamientos humanos como nunca antes habían podido observarse. Ahora bien, la cuestión planteada es la propiedad y los fines para los que se emplean estos datos. La realidad es que es poca la cantidad de datos a la que es posible acceder como investigador, 
pudiendo reducirse a algunas redes sociales. Las empresas de telefonía móvil, banca o compañías tecnológicas (Google, Facebook...) atesoran la gran masa de datos. El mundo académico y la administración pública tienen acceso muy limitado, ya que los beneficios asociados a esta información son enormes.

La secuencia se resume de la siguiente manera: (1) los datos los producen los usuarios de forma gratuita (2) las empresas que los almacenan y gestionan obtienen beneficios millonarios de su venta (el usuario se convierte en producto) (3) las empresas que compran esta información también mejoran sus ventas al enfocar su publicidad al público más adecuado (4) se incita a los usuarios a un mayor consumo, lo que implica un gasto que impacta en su propia economía.

Aceptando el hecho que el uso de las nuevas tecnologías suponga la mercantilización de nuestra intimidad, ¿no cabría la posibilidad de demandar una democratización en los medios de acceso a estos datos? Además de aumentar nuestro consumo, ¿sería posible que pudieran orientarse a conocer comportamientos humanos para que las políticas públicas incidieran en una mejora de la calidad de vida de los ciudadanos? Una legislación al respecto podría ser clave en este asunto.

Agradecimientos: Este artículo se ha elaborado en el marco del proyecto "Sostenibilidad social, conectividad global y economía creativa como estrategias de desarrollo en el Área metropolitana de Valencia" (CSO2016-74888-C4-1-R), financiado por la Agencia Estatal de Investigación (AEI) y el Fondo Europeo de Desarrollo Regional (FEDER) dentro del Programa Estatal de Investigación, Desarrollo e Innovación Orientada a los Retos de la Sociedad, incluido en el Plan Estatal de Investigación Científica y Técnica y de Innovación 2013-2016, convocatoria de 2016. Carmen Zornoza cuenta con una ayuda para contratos predoctorales para la formación de doctores (BES2014-067846) dentro del Subprograma Estatal de Formación del Programa Estatal de Promoción del Talento y su Empleabilidad, en el marco del Plan Estatal de Investigación Científica y Técnica y de Innovación 2013-2016, financiado por el Ministerio de Economía y Competitividad y cofinanciado por el Fondo Social Europeo.

Declaración responsable: La autora declara que no existe ningún conflicto de interés en relación a la publicación de este artículo. 


\section{Bibliografía}

Bretagnolle, A. (2009). Villes et réseaux de transport: des interactions dans la longue durée (France, Europe, Etats-Unis) (Mémoire d'habilitation à diriger des recherches, Université Paris 1 Panthéon-Sorbonne, UFR de Géographie, France). (Unpublished).

CEC, Commission of the European Communities (2004). Towards a Thematic Strategy on the Urban Environment. Brussels: 60 Final. Retrieved from http://www.europarl.europa.eu/meetdocs/committees/rett/20040316/com_com(2004)0060e $\underline{\text { n.pdf }}$

Charron, M. (2007). From excess commuting to commuting possibilities: More extension to the concept of excess commuting. Environment and Planning A, 39(5), 1238-1254. https://doi.org/10.1068/a34126

Duran, M. A. (2007). El valor del tiempo ¿ cuántas horas te faltan al día? Madrid: Espasa Calpe.

European Environmental Agency (2006). Urban Sprawl in Europe. The ignored challenge. Copenhagen. Retrieved from Retrieved from: https://www.eea.europa.eu/publications/eea_report_2006_10

European Environmental Agency (2018). Air quality in Europe - 2018 report. Retrieved from https: //www.eea.europa.eu/publications/air-quality-in-europe-2018

Generalitat Valenciana \& Conselleria de Medi Ambient, Aigua, Urbanisme i Habitatge (2011). Estrategia Territorial de la Comunidad Valenciana. Retrieved from http://www.habitatge.gva.es/es/web/planificacion-territorial-e-infraestructura-verde/estrategiaterritorial-de-la-comunitat-valenciana-77496

García-Paloma res, J. C., Salas-Olmedo, M. H., Moya-Gómez, B., Condeço-Melhorado, A. M., \& Gutiérrez, J. (2018). City dynamics through Twitter: relationships between land use and spatiotemporal demographics. Cities, 72, 310-319.

https://doi.org/10.1016/j.cities.2017.09.007

Gielen, E. (2016). Costes del 'Urban Sprawl' para la Administración local. El caso valenciano. Valencia: Universidad de València.

Goodchild, M. F. (2007). Citizens as sensors: The world of volunteered geography. Geofournal, 69(4), 211-221. https://doi.org/10.1007/s10708-007-9111-y

Gutiérrez, J., García-Palomares, J. C., Romanillos, G., \& Salas-Olmedo, M.H. (2017). The eruption of Airbnb in tourist cities: Comparing spatial patterns of hotels and peer-to-peer accommodation in Barcelona. Tourism Management, 62, 278-291.

https://doi.org/10.1016/j.tourman.2017.05.003 
Indovina, F. (1998). Algunes consideracions sobre la "ciutat difusa". Documents d'anàlisi geogràfica, 33, 21-3. Retrieved from

https://ddd.uab.cat/pub/dag/02121573n33/02121573n33p21.pdf

Jurdak, R., Zhao, K., Liu, J., Aboulaoude, M., Cameron, M., \& Newth, D. (2015). Understanding human mobility from Twitter. PloS one, 10(7). https://doi.org/10.1371/journal.pone.0131469

Kesselring, S. (2006). Pioneering mobilities: new pattern of movement and mobility in a new world, Environment and Planning, 38, 269-279. https://doi.org/10.1068/a37279

Li, S., Dragicevic, S., Anton, F., Sester, M., Winter, S., Coltekin, A., Pettit, C., Jiang, B., Haworth, J., \& Stein, A (2016). Geospatial big data handling theory and methods: A review and research challenges. Isprs journal of photogrammetry and remote sensing, 115, 119-133. https://doi.org/10.1016/j.isprsjprs.2015.10.012

Marchetti, C. (1994). Anthropological invariants in travel behavior. Technological Forecasting and Social Change, 47(1), 75-88. https://doi.org/10.1016/0040-1625(94)90041-8

Miralles-Guasch, C. (2002). Ciudad y Transporte. El binomio imperfecto. Barcelona: Ariel.

Merlin, P. (1991), Géographie, économie et planification des transports. Paris: PUF.

Miralles-Guasch, C. (2011). Dinámicas metropolitanas y tiempos de la movilidad. La región metropolitana de Barcelona, como ejemplo. Anales de Geografía de la Universidad Complutense, 31(1), 125-145. https://doi.org/10.5209/rev_AGUC.2011.v31.n1.6

Miralles-Guasch, C., \& Cebollada, A. (2009). Movilidad cotidiana y sostenibilidad una interpretación desde la geografía humana. Boletín de la Asociación de Geógrafos Españoles, 50, $193-216$

Munizaga, M., Palma, C., \& Mora, P. (2010). Public transport OD matrix estimation from smart card payment system data. In Proceedings from 12th World Conference on Transport Research. Lisbon: Paper, 2988. Retrieved from http://www.civil.ist.utl.pt/wctr12_lisboa/proceedings.htm

Næss, P. (2006). Urban structure matters. Residential location, car dependance and travel behaviour. New York: Taylor \& Francis. https://doi.org/10.1080/09640560701402133

Nel.lo, O. (1996). Els confins de la ciutat sense confins. Estructura urbana i límits administratius de la ciutat difusa. In A. Castañer, M. Falgueras \& J. Vicente (Eds.), La ciutat difusa i les perifèries: Experiències de planificació i gestió (pp. 55-72). Girona: Universitat de Girona.

Nel.lo, O. (2002). Cataluña, ciudad de ciudades. Lleida: Milenio

Newman, P., \& Kenworthy, J. R. (2006). Urban Design to Reduce Automobile Dependence. Opolis, 2(1), 35-52. Retrieved from https://escholarship.org/uc/item/2b76f089 
Norman, J., Maclean, H. L., Asce, M., \& Kennedy, C. A. (2006). Comparing High and Low Residential Density: Life-Cycle Analysis of Energy Use and Greenhouse Gas Emissions. Journal of Urban Planning and Development, 132(1), 10-21. https://doi.org/10.1061/(ASCE)07339488(2006)132:1(10)

OECD (2010). Cities and Climate Change. Paris: OECD Publishing. https://doi.org/10.1787/9789264091375-en

Pumain, D. (2003). Scaling laws and urban systems (Working Papers, $\left.n^{\circ} 4\right)$. Santa Fe Institute. Retrieved from https://sfi-edu.s3.amazonaws.com/sfi-edu/production/uploads/sficom/dev/uploads/filer/23/4d/234dc29d-faf5-4715-bd7e-a418c8cc9e9a/04-02-002.pdf

Rajamani, J., Bhat, C.R., Handy, S., Knaap, G., \& Song, Y. (2003). Assessing impact of urban form measures on nonwork trip mode choice after controlling for demographic and level-of-service effects. Transportation Research D, (1831), 158-165. https://doi.org/10.3141/1831-18

Salas-Olmedo, M. H., Moya-Gómez, B., García-Palomares, J. C., \& Gutiérrez, J. (2018). Tourists' digital footprint in cities: Comparing Big Data sources. Tourism Management, 66, 13-25. https://doi.org/10.1016/j.tourman.2017.11.001

Salom, J. y Albertos, J.M. (2010). Densidad de la red viaria y forma urbana: Delimitación del espacio urbano en ocho aglomeraciones españolas. In J. M. Feria Toribio \& J. M. Albertos Puebla (Eds.), La ciudad metropolitana en España: Procesos urbanos en los inicios del siglo XXI. Thomson Reuters, Pamplona (pp. 49-94).

Serra, A. (2007). Vida quotidiana en un espai urbà transformat. El Mercadal de Girona des d'una perspectiva de gènere. Documents. Anàlisis Geográfica, 49, 161-180. Retrieved from https://ddd.uab.cat/pub/dag/02121573n49/02121573n49p161.pdf

Schafer, A., \& Victor, D. (2000). The future mobility of the world population. Transportation Research Part A, 34(3), 171-205. http://dx.doi.org/10.1016/S0965-8564(98)00071-8

Tobler, W.R. (1970). A Computer Movie Simulating Urban Growth in the Detroit Region. Economic Geography, 46 (Supplement), 234-240.

Zahavi, Y. (1974). Travel time budgets and mobility in urban areas (Report prepared for the U.S. Department of Transportation and the Ministry of Transport of Federal Republic of Germany). Retrieved from http://www.surveyarchive.org/Zahavi/TravelTime_Budgets.pdf 